\title{
Retrieval of Quantitative and Qualitative Information about Plant Pigment Systems from High Resolution Spectroscopy
}

\author{
Susan L. Ustin ${ }^{1}$, Senior Member IEEE, Gregory P. Asner ${ }^{2}$, John A. Gamon ${ }^{3}$, K. Fred \\ Huemmrich $^{4}$, Stéphane Jacquemoud ${ }^{5}$, Michael Schaepman ${ }^{6}$, Member IEEE, Pablo Zarco-Tejada ${ }^{7}$ \\ ${ }^{1}$ Dept. L.A.W.R., University of California Davis 95616 (USA), p 001-530-752-0621, email: \\ slustin@ucdavis.edu, ${ }^{2}$ Carnegie Institution of Washington, Stanford, CA, USA, ${ }^{3}$ California State University \\ Los Angeles, CA, USA, ${ }^{4}$ NASA GSFC, Greenbelt, MA, USA, ${ }^{5}$ Institut de Physique du Globe de Paris, \\ ${ }^{6}$ Wageningen University, NL, ${ }^{7}$ Instituto de Agricultura Sostenible, Consejo Superior de Investigaciones \\ Científicas (ES)
}

\begin{abstract}
Life on earth depends on photosynthesis. Photosynthetic systems evolved early in earth history and have been stable for $\mathbf{2 . 5}$ billion years, providing prima facie evidence for these significance of evolutionary functions. Pigments perform multiple plant functions from increasing the range of energy captured for photosynthesis to a range of protective functions. Given the importance of pigments to leaf functioning, greater effort is needed to determine whether individual pigments can be identified and quantified by high fidelity spectroscopy. New methods to identify overlapping pigment absorptions would provide a major advance for understanding plant functions, quantifying net carbon exchange, and identifying plant stresses.
\end{abstract}

Keywords-plant pigments, chlorophyll a, b, carotenes, xanthophyll, leutin, anthocyanin pigments, absorption features, spectral measures of pigments

\section{INTRODUCTION}

Life on Earth is driven by photosynthesis, producing both oxygen and organic matter [1]. Photosynthesis is one of the earliest biological processes and the pigment systems in modern photosynthetic bacteria, algae, and plants appeared early in Earth's history, at least 2.5 billion years [2]. Photosynthetic pigments of modern photosynthetic bacteria, algae, and plants, including chlorophyll $a, b$, and various carotene pigments, date from this period. The length of this record and its stability demonstrate the functional importance of these photosynthetic pigments and the rationale for remotely measuring them. In fact, the stability of chlorophyll molecules make them a target, along with water, in the search for extraterrestrial life [3].

The light reactions of photosynthesis are driven by four multi-subunit membrane protein complexes named photosystem I, photosystem II, cytochrome b6f complex and the F-ATPase complex [4]. In the intact chloroplast, pigment-protein complexes are associated with grana or stroma lamellae membranes. PS I and II contain chlorophyll and other pigments that harvest light and transfer energy to the reaction centers, which are composed of a single chlorophyll $a$ molecule. It is known that the two photosystems differ in chlorophyll $a$ concentration, with approximately $10 \%$ more chlorophyll associated with PSI. Two distinct photosystem subtypes occur in both PSI and PSII [1] which differ in the number of chlorophyll molecules in the antenna. Major pigments also include $\beta$ carotene, lutein and xanthophyll cycle pigments [5]. The size and composition of the pigments in the photosynthetic antenna associated with each reaction center is flexible depending on environmental conditions.

Both eukaryotic organisms and prokaryotic bluegreen bacteria have nearly identical in subunit composition of photosystems I and II including the reaction centers [1]. These lines of evidence support the evolutionary importance of both the pigment composition and chloroplast structure for biophysical functioning. In recent years plant physiologists and geneticists have greatly extended our knowledge of the three dimensional structure and mechanisms of the pigment complexes in chloroplasts and the genetic inheritance of subcomponents. Developing the methods to quantify pigment composition and concentration from remotely sensed data would clearly provide an advance in understanding photosynthetic processes and provide insight into detection of plant stresses.

\section{SPECTROSCOPY}

From the beginning of systematic earth observation, remote sensing has focused on measuring plant pigments, often described as synonymous with chlorophyll content or even simply "greenness". There 
is a close relationship between photosynthetic capacity, estimated by pigment concentrations, and net primary production that is captured to first approximation by the chlorophyll absorption measured by broad-band multispectral imagers [6], [7], [8], [9]. That photosynthetic pigment absorptions (at least total pigments or chlorophyll) can be measured by remote sensing systems is well known [10], [11], [12], [13].

However, besides chlorophyll $a$ and $b$, photosynthetic reaction centers in cyanobacteria, eukaryotic algae, and plants contain other membranebound accessory pigments, alter the efficiency of photon capture and/or provide protective functions that avoid damage to the reaction center. For example, under full sunlight, light intensity may exceed the capacity for electron transfer between photosystems and some carotenoid pigments, such as xanthophylls, are involved with protection from photooxidation and photoinhibition. The size and composition of the pigments in the photosynthetic antenna associated with each reaction center is flexible depending on environmental conditions.

Remarkably, given the roles of accessory pigments in light capture, photosystem protection, and in various growth and development functions, they have received little attention by the remote sensing research community. This is largely attributed to the limited availability and/or data quality of the current generation of imaging spectrometers. Because pigments have distinctive absorption spectra depending on their molecular structure and local chemical environment, there is a potential to measure these properties using high fidelity reflectance spectroscopy.

Organisms grown in low light typically have more antenna pigments per reaction center than when grown at high light. Under high light the protective mechanism of the xanthophyll cycle pigments is well established [14], [15] There is strong evidence for optimization of efficiency at low light while avoiding photooxidation under high light conditions [16], [18], [17], observed short-term changes in reflectance near $530 \mathrm{~nm}$ that are detecting reversible changes in the distribution of xanthophyll cycle pigments in response to the light environment.

Both climatological and biogeochemical stressors are reported to increase the proportion of chlorophyll $b$ relative to chlorophyll $a$ [19]. Seasonal shifts of the long-wavelength edge of the chlorophyll absorption also have been observed [10], [20] and environmental stress related shifts [21], [22]. Chlorosis increases reflectance and causes a blue-shift due to narrowing and reduction in depth of the absorption feature [23]. Autumn colors result from the breakdown of chlorophyll pigments preceeding breakdown of carotenoid pigments.
Anthocyanins are red water-soluble nonphotosynthetic pigments that occur widely in flowers, fruits and leaves. They have a single absorption maximum around $529 \mathrm{~nm}$ and can be detected by reflectance changes in the red region [24], [25], [26]. Anthocyanins may function in protecting the photosynthetic system from excess light, particularly excess UV radiation, and they may be involved in protection from herbivory. Anthocyanins are often observed under environmental stresses (e.g., high temperatures) or during early leaf development [27], consequently, their measurement may provide another indicator of physiological state.
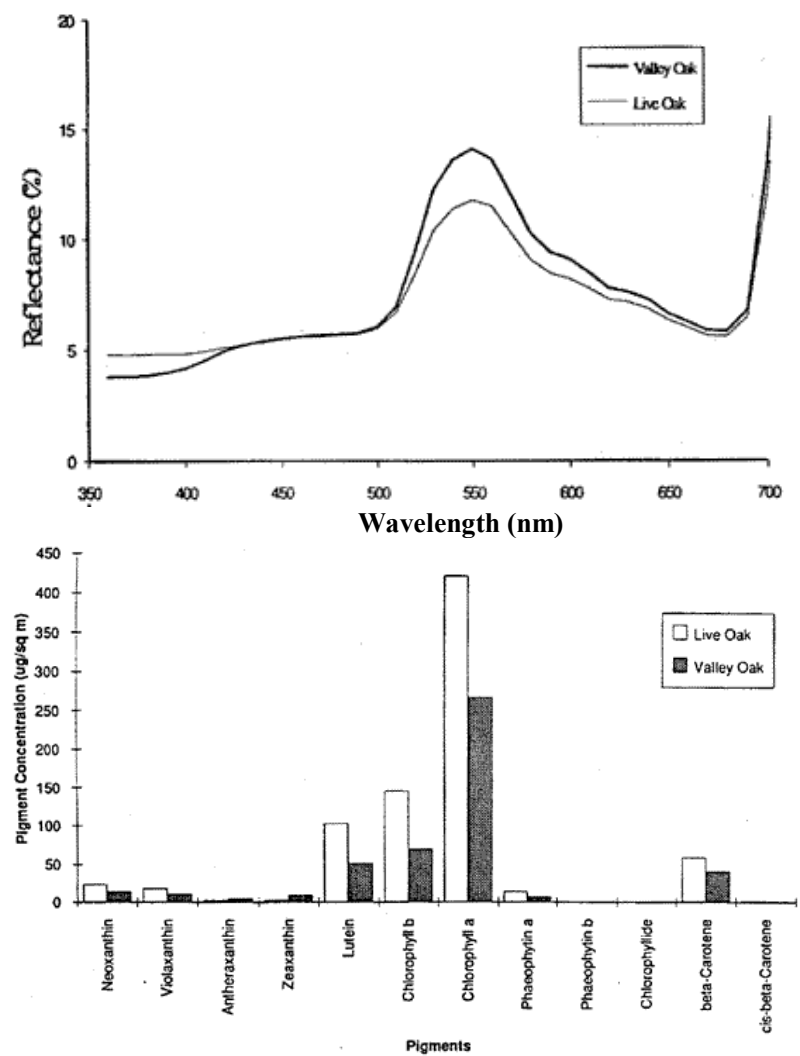

Figure 1. (upper) Mean reflectance spectra of two species of California oak (Quercus), measured in mid-summer at Jasper Ridge Biological Preserve. (lower) Mean pigment concentration for these species.

With today's high fidelity imaging spectroscopy, it is possible to measure small absorption features that can capture variations in pigment composition and concentration. Despite the importance of accessory pigments in photosynthesis and decades of remote sensing of leaf and canopy "greenness", the ability to identify and quantify plant pigments remains underutilized. We review existing evidence for detection and quantification of photosynthetic pigments and methods of analysis. 
There is strong interest today in developing and validating techniques to detect and quantify individual pigments that can advance understanding of biophysical functioning in plants. Light absorption by photosynthetic pigments dominates green leaf properties in the visible spectrum $(400-700 \mathrm{~nm})$, whereas more general photon-matter interaction with specific absorption features in leaves can be found throughout the solar reflected wavelength range (400$2500 \mathrm{~nm}$ ). At least 25 spectral indexes, many designed to take advantage of narrow spectral bands, have been used to measure leaf and plant pigments. We will first review the use of quantitative statistical based methods with examples and evaluate their strengths and weaknesses for identifying and quantifying individual pigments and states, including fluorescence emissions. One explanation for past inconsistency in separating and quantifying different pigments is because of their overlapping absorption spectra. Indexes and other simple methods do not account for the interacting effects of multiple pigments. In some cases, indexes that seem to provide consistent results at the leaf level fail or become inconsistent at the canopy or stand levels, in part due to the complexities of threedimensional structure and multiple scattering.

Leaf optical properties models, e.g., PROSPECT, LIBERTY, LEAFMOD, SLOP [23] also have been used to predict chlorophyll concentration, assuming that leaves are entirely composed of chlorophyll pigments. Extending radiative transfer models to detect other pigment molecules, e.g., different xanthophyll and carotene pigments or even separation of chlorophyll $a$ and $b$ is needed. These models require in vivo absorption coefficients for individual pigments which are not currently available, and which may vary depending upon the chemical environment within the leaf. The overlapping wavelengths of these absorption spectra make individual identification challenging and more work is needed to understand the impact of nested absorption feature on others. We will review the state of existing models and opportunities for improving the range of biochemicals detected.

It has long been noted that extracted chlorophyll absorption peaks are shifted about $20 \mathrm{~nm}$ to shorter wavelengths than observed from leaf reflectance. In intact leaves, both a blue shift of red edge first reported by [10] and later by [28] and reported red shifts are due to differences in chlorophyll concentration. Despite 30 years of work, defining the relationship between the red edge and chlorophyll concentration remains an area of active research [29], [30]. This may be due to difficulty in measuring the suite of pigments and intermediates and any conformational changes. [31] found a broadening of the chlorophyll absorption band in Ponderosa pine needles with atmospheric ozone exposure which mimicked increased chlorophyll concentration, possibly due to an increase in the disorder of the absorbing medium, a pattern consistent with observations that an early sign of ozone injury is granulation of the thylakoid stroma in chloroplasts. Using combined LIBERTY and SAIL, (LIBSAIL), [32] designed a chlorophyll index for MERIS based on selected wavebands of the red edge.

To date, astrophysicists have detected the existence of about 180 exoplanets. The search of possible life in the form of vegetation using the red edge is a challenge which has excited them for a few years [33], [34], [35].

\section{NEXT STEPS}

We think three lines of evidence are needed to advance current capabilities. First, investigate empirical correlations with pigment concentrations and reflectance patterns. Identification and quantification of individual pigments have been tried in various vegetation indexes currently available with partial success. New approaches that model the effect of one absorption feature on another are needed (e.g., multiple Gaussian models developed by [36]. Second, advances in radiative transfer models like PROSPECT and SAIL by developing new absorption coefficients for additional pigments is needed. And third, experimental approaches performed on intact leaves that will advance understanding of photosynthetic functioning and light responses, which would be best performed in collaboration with plant anatomists and physiologists. Lastly, leaf fluorescence could provide additional critical information about photosynthetic function. Recent studies of diurnal and long-term water stresses [37], [38] demonstrate that steady-state chlorophyll $a$ fluorescence is detectable under controlled conditions from plant canopy reflectance.

\section{REFERENCES}

[1] N. Nelson and C.F. Yocum, "Structure of function of photosystems I and II," Annu. Rev. Plant Biol., vol. 57, pp.44, 2006.

[2] J.M. Olson and R.E. Blankenship, "Thinking about the evolution of photosynthesis," Photosynth. Res., vol. 80, pp. 373386, 2004.

[3] J.A. Raven and R.D. Wolstencroft, "Constraints on photosynthesis on Earth and Earth-like planets," IAU Symposia, vol. 213, pp. 305-308, 2004.

[4] N. Nelson and A. Ben-Shem, "The structure of photosystem I and evolution of photosynthesis," BioEssays, vol. 27, pp. 914922, 2005 .

[5] H.K. Lichtenthaler, "Cholrophyll fluorescence signatures of leaves during the autumnal chlorophyll breakdown," J. Plant Physiol., vol. 131, pp. 101-110, 1987.

[6] G. Asrar, H. Fuchs, E.T. Kanemasu, J.L. Hatfield, "Estimating absorbed photosynthetic radiation and leaf-area index from spectral reflectance in wheat," Agronomy J. vol. 76, pp. 300306,1984

[7] J.L. Monteith, Vegetation and the Atmosphere. Academic Press, New York, vol. 2, 1976. 
[8] P.J. Sellers, "Canopy reflectance, photosynthesis and transpiration,” Int. J. Remote Sens., vol. 6, pp.1335-1372, 1985.

[9] P.J. Sellers, "Canopy reflectance, photosynthesis, and transpiration. 2. The role of biophysics in the linearity of their interdependence," Remote Sens. Environ., vol. 21, pp. 143-183, 1987.

[10] D.M. Gates, H.J. Keegan, J.C. Schleter, V.R. Weider, "Spectral properties of plants," Applied Optics, vol. 4, pp. 11-20, 1965.

[11] D.M. Gates, "Physical and physiological properties of plants," in Remote Sensing with Special Reference to Agriculture and Forestry, National Research Council Committee on Remote Sensing for Agricultural Purposes, , Washington D.C.: National Academy of Sciences, 1970, pp. 224-252.

[12] H.W. Gausman, "Reflectance of leaf components," Remote Sens. Environ., vol. 6, pp. 1-9, 1977.

[13] H.W. Gausman, D.E. Escobar, J.H. Everitt, A.J. Richardson, and R.R, Rodriguez, "Distinguishing succulent plants from crop and woody-plants," Photogram. Eng. Remote Sens., vol. 44, pp. 487-491, 1978.

[14] B. Demmig-Adams and W.W. Adams, "The role of xanthophyll cycle in the protection of photosynthesis," Trends in Plant Science, vol. 1, pp. 20-26, 1996.

[15] B. Demmig-Adams and W.W. Adams, 1996b.

[16] P. Horton, A.V. Ruban, and R.G. Walters, "Regulation of Light Harvesting in Green Plants," Plant Physiol., vol. 106c, pp. 415420, 1994.

[17] S. Bailey, R. Walters, S. Jansson, and P. Horton, "Acclimation of Arabidopsis thaliana to the light environment: the existence of separate low light and high light responses," Planta, vol. 213, pp. 794-801, 2001.

[18] J.A. Gamon, C.B. Field, W. Bilger, O. Bjorkman, A.L. Fredeen, J. Penuelas' "Remote-sensing of the xanthophylls cycle and chlorophyll fluorescence in sunflower leaves and canopies," Oecologia, vol. 85, pp. 1-7, 1990.

[19] J Anderson, W. Chow and D. Goodchild, "Thylakoid membrane organisation in sun/shade acclimation," Australian J. Plant Physiol., vol. 15, pp. 11-26, 1988.

[20] D.N. Horler, M. Dockray, and J. Barber, "The red edge of plant leaf reflectance," Int. J. Remote Sens., vol. 4, pp. 273-288, 1983.

[21] S.H. Chang and W. Collins, "Confirmation of the airborne biogeophysical mineral exploration technique using laboratory methods," Economic Geology, vol. 78, pp. 723-726, 1983.

[22] N.M. Milton, W. Collins, S-H. Chang, R.G. Schmidt, "Remote detection of metal anomalies on Pilot Mountain, Randolph County, North Carolina," Economic Geology, vol. 78, pp. 605$617,1983$.

[23]S.L. Ustin, S. Jacquemoud, P. Zarco-Tejada, and G. Asner, "Remote Sensing of Environmental Processes: State of the Science and New Directions," in Manual of Remote Sensing Vol. 4. Remote Sensing for Natural Resource Management and Environmental Monitoring. S.L. Ustin, vol. Ed. ASPRS. New York: John Wiley and Sons, 2004, pp. 679-730.

[24] P.J. Curran, W.R. Windham, H.L. Gholz, "Exploring the relationship between reflectance red edge and chlorophyll concentration in splash pine leaves," Tree Physiol., vol. 15, pp. 203-206, 1995.

[25] Neill S. and Gould K.S. (1999), Optical properties of leaves in relation to anthocyanin concentration and distribution, Canadian Journal of Botany, 77(12):1777-1782.

[26] Gitelson A.A., Merzlyak M.N. and Chivkunova O.B. (2001), Optical properties and nondestructive estimation of anthocyanin content in plant leaves, Photochemistry and Photobiology, 74(1):38-45

[27] D.A. Sims and J.A. Gamon, "Relationships between leaf pigment content and spectral reflectance across a wide range of species, leaf structures, and developmental stages," Remote Sens. Environ., vol. 81, pp. 337-354, 2002.

[28] W. Collins, "Remote sensing of crop type and maturity," Photogram. Eng. Remote Sens., vol. 44, pp. 43-55, 1978
[29] P.J. Curran, J.L. Dungan, B.A. Macler, and S.E. Plummer, "The effect of a red pigment on the relationship between red edge and chlorophyll concentration," Remote Sens. Environ., vol. 35, pp. 69-76, 1991.

[30] A. Pinar and P.J. Curran, "Grass chlorophyll and the reflectance red edge," Int. J. Remote Sens., vol. 17, pp. 351-357, 1996.

[31] B. Curtiss and S.L. Ustin, "Parameters affecting reflectance of coniferous forests in the region of chlorophyll pigment absorption," in IGARSS '89 Proc. Int. Geosci. Remote Sens. Symp. Vancouver, BC, Canada. July, 1989. IEEE 89CH27680, vol. 4, pp. 2633-2636.

[32] J. Dash and P.J. Curran, MTCI: The MERIS terrestrial chlorophyll index, in Proc. MERIS User Workshop, Frascatti (Italy), ESA, Vol. SP-549, pp. 11, 2003

[33] L. Arnold., S. Gillet, O. Lardiere, P. Riaud and J. Schneider, A test for the search for life on extrasolar planets, Astronomy \& Astrophysics, 392:231-237, 2002.

[34] S. Seager, E.L. Turner, J. Schafer and E. B. Ford, Vegetation's red edge: a possible spectroscopic biosignature of extraterrestrial plants, Astrobiology, 5(3):372-390, 2005.

[35] G. Tinetti, S. Rashby and Y.L. Yung, Detectability of red-edge shifted vegetation on terrestrial planets orbiting M-stars, unpublished.

[36] J.M. Sunshine, C.M. Pieters, \& S.F. Pratt, Deconvolution of mineral absorption bands - an improved approach Journal of Geophysical Research-Solid Earth and Planets, 95, 6955-6966, 1990.

[37] P.J. Zarco-Tejada, J.C. Pushnik, S. Dobrowski, and S.L. Ustin, Steady-state chlorophyll fluorescence detection from canopy derivative reflectance and double-peak effects. Remote Sensing of Environment 84(2): 283-294, 2003.

[38] S.Z. Dobrowski, J.C. Pushnik, P.J. Zarco-Tejeda, and S.L. Ustin. Simple reflectance indices track heat and water stressed induced changes in steady state chlorophyll fluorescence at the canopy scale. Remote Sensing of Environment 97(3): 403-414, 2005 . 